\title{
New records of the rare Troschel's Pampas Snake, Phimophis guianensis (Serpentes: Dipsadidae) in Brazil
}

\author{
OMAR M. ENTIAUSPE-NETO ${ }^{1}$, WEVERTON S. AZEVEDO ${ }^{2}$, VALQUIRIA O. PEREIRA ${ }^{2}$, \\ ARTHUR D. ABEGG ${ }^{2}$, ANDERSON M. ROCHA ${ }^{3}$ and DANIEL LOEBMANN ${ }^{1}$ \\ ${ }^{1}$ Universidade Federal do Rio Grande, Instituto de Ciências Biológicas, Laboratório de \\ Vertebrados, Avenida Itália, Km 8, Vila Carreiros, 96203-900 Rio Grande, RS, Brazil \\ ${ }^{2}$ Instituto Butantan, Laboratório Especial de Coleções Zoológicas, Avenida \\ Vital Brasil, 1500, Butantã, 05503-900 São Paulo, SP, Brazil \\ ${ }^{3}$ Faculdade Cathedral de Ensino Superior, Laboratório de Zoologia Aplicada de Vertebrados Terrestres \\ e Aquáticos, Avenida Luis Canuto Chaves, 293, Caçari, 69307-655 Boa Vista, RR, Brazil \\ Manuscript received on February 7, 2018; accepted for publication on April 10, 2018
}

\begin{abstract}
The Troschel's Pampas Snake, Phimophis guianensis (Troschel, 1848), is widely distributed in Amazonian Savannas at northern South America and a small portion of southern Central America, being recorded to Brazil based on three historical records, that ranged from 1997 to 2002, in Amapá and Pará states. In this study, we revise all known records of P. guianensis, providing an updated distribution map, and the first record to Roraima state.
\end{abstract}

Key words: Amazonian Savannah, Roraima, distribution gap, Squamata, Pseudoboini.

\section{INTRODUCTION}

Amazonian Savannas are sparsely distributed and fragmented across northern South America, being characterized by a unique phytophysiognomy of arbustive and grassland plant species, that contrast with the tall-canopy tropical rainforest that encircle it (Eiten 1978, França et al. 2006, Carvalho et al. 2016). It has also been suggested that these grassland enclaves within the Amazon forest constitute relictual portions of a once wide savanna formation, that encompassed most of northern South America, southwards to central Brazil, and rose during the Pleistocene epoch, as the outcome

Correspondence to: Omar Machado Entiauspe-Neto

E-mail: omarentiauspe@hotmail.com of glacial periods (Eden 1974, Ab'Sáber 1982, Bigarella and Andrade-Lima 1982, Huber 1982, França et al. 2006). These areas usually present poorly diverse, although highly endemic reptile communities, that have been seldom studied (Ávila-Pires 1995, Vitt and Carvalho 1995, Colli 1996, França et al. 2006).

Phimophis Cope 1860 is a Pseudoboini genus that encompasses three species, Phimophis guerini (Duméril, Bibron, and Duméril 1854), Phimophis guianensis (Troschel 1848) and Phimophis vittatus (Boulenger 1896), distributed from Central America, in Panama, to South America, in Argentina and southern Brazil (Peters et al. 1970, Uetz et al. 2017). These are terrestrial or fossorial, nocturnal, small 
to medium sized snakes, that prey upon lizards, amphibians and rodents (Yanosky et al. 1996, Sawaya et al. 2008, González-Carcacía et al. 2012).

The Troschel's Pampas Snake, Phimophis guianensis (Troschel 1848), has been recorded from Panamá, in Central America, to Colombia, Venezuela, Guyana, Surinam, French Guyana, and Brazil (Troschel 1848, Dunn 1944, Hoogmoed 1982, Lancini and Kornacker 1989, Frota et al. 2005, França et al. 2006, Cole et al. 2013, BlancoTorres et al. 2013). Phimophis guianensis was first recorded in Brazil based on three specimens from Amazonian Savanna areas at Amapá and Pará states, in northern Brazil, that ranged from a timespan of 1997 to 2002 (Frota et al. 2005 and França et al. 2006). Herein, we provide new records of this species at Brazilian territory.

\section{MATERIALS AND METHODS}

While conducting fieldwork in the municipality of Cantá (2.2000N, -60.4833W, DATUM WGS 84), Roraima State, Brazil (Figure 1), on 28 April 2017, at 22:25 hours, the authors encountered two individuals of $P$. guianensis, shortly apart from each other, at the Km 16 of the BR-401. Identification follows Starace (1998) and Mumaw et al. (2015). Tissue samples were deposited in $90 \%$ ethanol, and individuals were fixated in a solution of $10 \%$ formalin, then preserved in $75 \%$ ethanol, and deposited in the herpetological collection of Universidade Federal do Rio Grande (Rio Grande, Rio Grande do Sul, Brazil) under the voucher CHFURG 5888 and CHFURG 5889. Measurements were taken with a flexible ruler or a dial caliper. SVL refers to "snout-vent length", TL

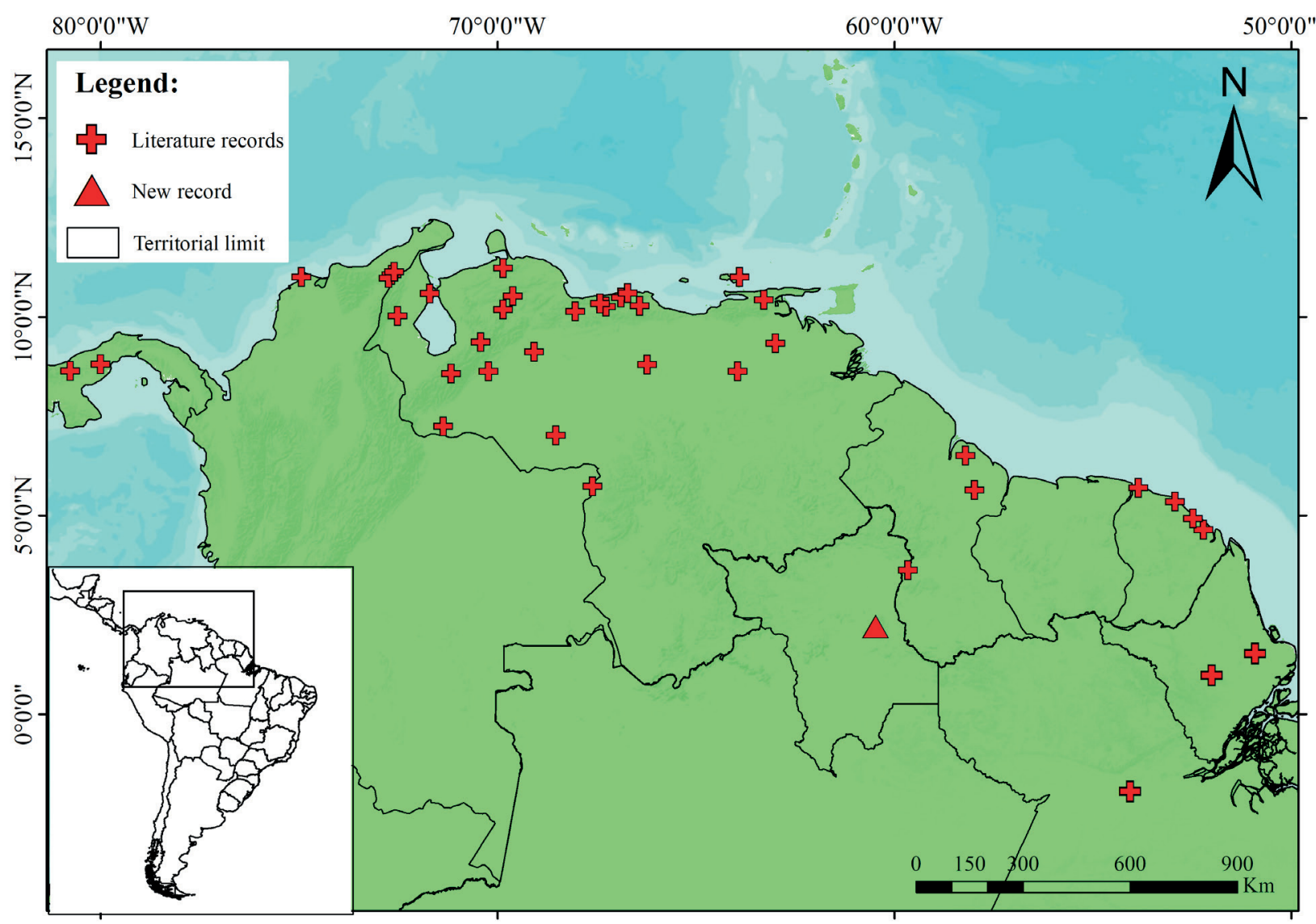

Figure 1 - Distribution of Phimophis guianensis (Triangle: new records; Crosses: literature records). 
to "tail length", HL to "head length", and HW to "head width".

\section{RESULTS}

The specimen CHFURG 5889 is a subadult male (Fig. 2a), that presents $350 \mathrm{~mm}$ snout-vent length, $120 \mathrm{~mm}$ tail length, $10.5 \mathrm{~mm}$ head length and 8.9 $\mathrm{mm}$ head width. Scale counts are 164 ventrals, 56 paired subcaudals, divided nasal scale, $8 / 8$ supralabials, $7 / 8$ infralabials, $2+3 / 2+3$ temporals, 1 preocular and $1+1$ postoculars. Specimen CHFURG 5888 is a subadult male (Fig. 2b), that presents 335 $\mathrm{mm}$ snout-vent length, $80 \mathrm{~mm}$ tail length, $9.2 \mathrm{~mm}$ head length, $7.4 \mathrm{~mm}$ head width. Scale counts are 169 ventrals, 56 paired subcaudals, divided nasal scale, 8/8 supralabials, 8/9 infralabials, $1+1$ fused temporals, 1 preocular and $1+1$ postoculars.

In life, both individuals presented a white supralabial and gular region, with a a black stripe that extended 14 dorsal scale rows from the head, with grey scale margins on the head surface; dorsal coloration composed of a irregular black pattern, over an orange background, with a white dorsolateral surface. Ventral coloration immaculate white on both specimens.

\section{DISCUSSION}

Phimophis guianensis (Troschel 1848) was first recorded to Brazil by Frota et al. (2005), based on a specimen from Monte Alegre (CHUNB 33929), Pará state, northern Brazil. França et al. (2006), seemingly unaware of the work of Frota et al. (2005), presented three new individuals for Amapá (CHUNB 03824-5, locality given as "Amapá, 22.vii.1997"; CHUNB 33929, locality given as "Tartarugalzinho, 02.v.1997"), claiming these, erroneously, as the first record to Brazil of the species. Since then, no new records arose from highly sampled areas (e.g. Thomas 1976, Cunha and Nascimento 1980, 1993, Hoogmoed 1982, Cunha et al. 1985, Vanzolini 1986, Zaher 1996, Vanzolini and Calleffo 2002), and this

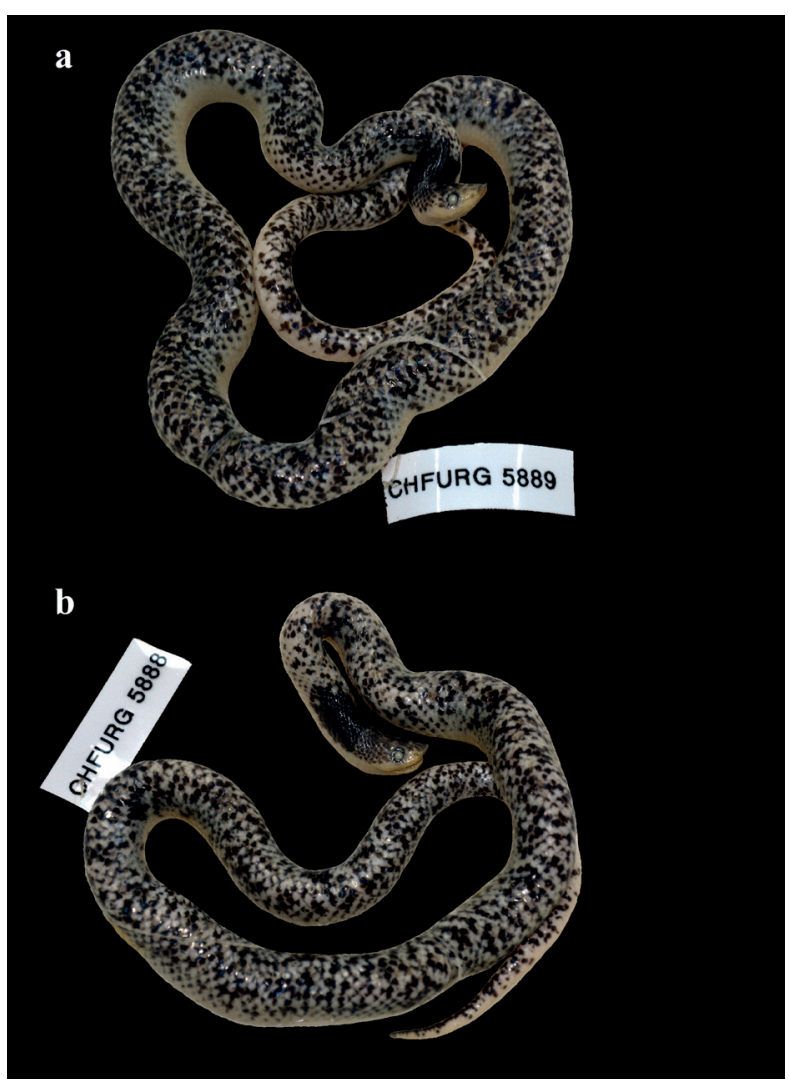

Figure 2 - Phimophis guianensis individuals found in the municipality of Cantá, Roraima.

species has been only known based on specimens from outside Brazil (Table I), which corroborates the hypothesis that this species might be rare in Brazil (França et al. 2006). The individuals from Cantá here described represent new records to Brazil, filling a distribution gap of approximately $140 \mathrm{~km}$ southward from the record of Pirara, Sabana, Guyana, and 882 $\mathrm{km}$ northward from the record of Monte Alegre, Pará, Brazil, and the first record of the species to Roraima state. These specimens also fit the literature diagnosis of the species (Troschel 1848, Starace 1998, Gaiarsa et al. 2013, Mumaw et al. 2015).

Roraima state is located in the northern portion of the Brazilian Amazon, presenting an area of $224.299 \mathrm{~km}^{2}$ (Barbosa and Lima 2008, Carvalho et al. 2016), of which nearly $20 \%$ is composed of savannas (Flores 2014), being these savannas largely composed of "lavrado" (Morais and 
TABLE I

Geographic distribution of Phimophis guianensis.

\begin{tabular}{|c|c|c|c|c|c|}
\hline Country & State/departament & Municipality/locality & Latitude & Longitude & Source \\
\hline Brazil & Amapá & Amapá & $00^{\circ} 58^{\prime} \mathrm{N}$ & $52^{\circ} 00^{\prime} \mathrm{W}$ & França et al. (2006) \\
\hline Brazil & Amapá & Tartarugalzinho & $01^{\circ} 31^{\prime} \mathrm{N}$ & $50^{\circ} 54^{\prime} \mathrm{W}$ & França et al. (2006) \\
\hline Brazil & Pará & Monte Alegre & $01^{\circ} 57^{\prime} \mathrm{S}$ & $54^{\circ} 03^{\prime} \mathrm{W}$ & $\begin{array}{l}\text { Frota et al. (2005), França et al. } \\
\qquad(2006)\end{array}$ \\
\hline Brazil & Roraima & Cantá & $02^{\circ} 12^{\prime} \mathrm{N}$ & $60^{\circ} 28^{\prime} \mathrm{W}$ & New record \\
\hline Colombia & La Guajira & Cerrejón & $11^{\circ} 03^{\prime} \mathrm{N}$ & $72^{\circ} 40^{\prime} \mathrm{W}$ & Blanco-Torres et al. (2013) \\
\hline Colombia & La Guajira & Palomino & $10^{\circ} 58^{\prime} \mathrm{N}$ & $72^{\circ} 45^{\prime} \mathrm{W}$ & Blanco-Torres et al. (2013) \\
\hline Colombia & La Guajira & Río Ranchería & $11^{\circ} 07^{\prime} \mathrm{N}$ & $72^{\circ} 36^{\prime} \mathrm{W}$ & Blanco-Torres et al. (2013) \\
\hline Colombia & Atlántico & Puerto Colombia & $11^{\circ} 00^{\prime} \mathrm{N}$ & $74^{\circ} 57^{\prime} \mathrm{W}$ & Dugand and Toloza (1975) \\
\hline Guiana & Demerara-Mahaica & $\begin{array}{l}\text { Ceiba Biological Station, } \\
\text { Madewini River }\end{array}$ & $06^{\circ} 29^{\prime} \mathrm{N}$ & $58^{\circ} 13^{\prime} \mathrm{W}$ & Cole et al. (2013) \\
\hline Guiana & $\begin{array}{l}\text { Alto Demerara- } \\
\text { Berbice }\end{array}$ & Dubulay Ranch & $05^{\circ} 38^{\prime} \mathrm{N}$ & $57^{\circ} 59^{\prime} \mathrm{W}$ & Cole et al. (2013) \\
\hline Guiana & Pirara & Sabana & $03^{\circ} 37^{\prime} \mathrm{N}$ & $59^{\circ} 40^{\prime} \mathrm{W}$ & Mumaw et al. (2015) \\
\hline $\begin{array}{l}\text { French } \\
\text { Guiana }\end{array}$ & Mana & - & $05^{\circ} 41^{\prime} \mathrm{N}$ & $53^{\circ} 52^{\prime} \mathrm{W}$ & Starace (1998) \\
\hline $\begin{array}{l}\text { French } \\
\text { Guiana }\end{array}$ & Roura & - & $04^{\circ} 38^{\prime} \mathrm{N}$ & $52^{\circ} 13^{\prime} \mathrm{W}$ & Starace (1998) \\
\hline $\begin{array}{l}\text { French } \\
\text { Guiana }\end{array}$ & Cayenne & Caiena & $04^{\circ} 55^{\prime} \mathrm{N}$ & $52^{\circ} 29^{\prime} \mathrm{W}$ & $\begin{array}{c}\text { Chippaux (1986), França et al. } \\
\text { (2006) }\end{array}$ \\
\hline $\begin{array}{l}\text { French } \\
\text { Guiana }\end{array}$ & Cayenne & Sinnamary & $05^{\circ} 20^{\prime} \mathrm{N}$ & $52^{\circ} 56^{\prime} \mathrm{W}$ & $\begin{array}{c}\text { Chippaux (1986), França et al. } \\
\text { (2006) }\end{array}$ \\
\hline Panama & Panamá & - & $08^{\circ} 37^{\prime} \mathrm{N}$ & $80^{\circ} 46^{\prime} \mathrm{W}$ & Ray and Ruback (2015) \\
\hline Panama & Panamá Oeste & - & $08^{\circ} 48^{\prime} \mathrm{N}$ & $80^{\circ} 00^{\prime} \mathrm{W}$ & Ray and Ruback (2015) \\
\hline Venezuela & Amazonas & - & $05^{\circ} 43^{\prime} \mathrm{N}$ & $67^{\circ} 37^{\prime} \mathrm{W}$ & Mumaw et al. (2015) \\
\hline Venezuela & Anzoátegui & - & $08^{\circ} 37^{\prime} \mathrm{N}$ & $63^{\circ} 57^{\prime} \mathrm{W}$ & Mumaw et al. (2015) \\
\hline Venezuela & Apure & - & $07^{\circ} 00^{\prime} \mathrm{N}$ & $68^{\circ} 32^{\prime} \mathrm{W}$ & Mumaw et al. (2015) \\
\hline Venezuela & Aragua & - & $10^{\circ} 15^{\prime} \mathrm{N}$ & $67^{\circ} 16^{\prime} \mathrm{W}$ & Mumaw et al. (2015) \\
\hline Venezuela & Bolívar & - & $10^{\circ} 19^{\prime} \mathrm{N}$ & $67^{\circ} 25^{\prime} \mathrm{W}$ & Mumaw et al. (2015) \\
\hline Venezuela & Carabobo & - & $10^{\circ} 07^{\prime} \mathrm{N}$ & $68^{\circ} 2^{\prime} \mathrm{W}$ & Mumaw et al. (2015) \\
\hline Venezuela & Distrito Capital & - & $10^{\circ} 28^{\prime} \mathrm{N}$ & $66^{\circ} 54^{\prime} \mathrm{W}$ & Mumaw et al. (2015) \\
\hline Venezuela & Falcón & - & $11^{\circ} 13^{\prime} \mathrm{N}$ & $69^{\circ} 52^{\prime} \mathrm{W}$ & Mumaw et al. (2015) \\
\hline Venezuela & Guárico & - & $08^{\circ} 47^{\prime} \mathrm{N}$ & $66^{\circ} 14^{\prime} \mathrm{W}$ & Mumaw et al. (2015) \\
\hline Venezuela & Lara & - & $10^{\circ} 10^{\prime} \mathrm{N}$ & $69^{\circ} 52^{\prime} \mathrm{W}$ & Mumaw et al. (2015) \\
\hline Venezuela & Mérida & - & $08^{\circ} 34^{\prime} \mathrm{N}$ & $71^{\circ} 10^{\prime} \mathrm{W}$ & Mumaw et al. (2015) \\
\hline
\end{tabular}


TABLE I (continuation)

\begin{tabular}{|c|c|c|c|c|c|}
\hline Country & State/departament & Municipality/locality & Latitude & Longitude & Source \\
\hline Venezuela & Miranda & - & $10^{\circ} 16^{\prime} \mathrm{N}$ & $66^{\circ} 25^{\prime} \mathrm{W}$ & Mumaw et al. (2015) \\
\hline Venezuela & Monagas & - & $09^{\circ} 19^{\prime} \mathrm{N}$ & $63^{\circ} 0^{\prime} \mathrm{W}$ & Mumaw et al. (2015) \\
\hline Venezuela & Nueva Esparta & - & $11^{\circ} 00^{\prime} \mathrm{N}$ & $63^{\circ} 54^{\prime} \mathrm{W}$ & Mumaw et al. (2015) \\
\hline Venezuela & Portuguesa & - & $09^{\circ} 06^{\prime} \mathrm{N}$ & $69^{\circ} 05^{\prime} \mathrm{W}$ & Mumaw et al. (2015) \\
\hline Venezuela & Sucre & - & $10^{\circ} 25^{\prime} \mathrm{N}$ & $63^{\circ} 17^{\prime} \mathrm{W}$ & Mumaw et al. (2015) \\
\hline Venezuela & Trujillo & - & $09^{\circ} 21^{\prime} \mathrm{N}$ & $70^{\circ} 26^{\prime} \mathrm{W}$ & Mumaw et al. (2015) \\
\hline Venezuela & Vargas & - & $10^{\circ} 35^{\prime} \mathrm{N}$ & $66^{\circ} 44^{\prime} \mathrm{W}$ & Mumaw et al. (2015) \\
\hline Venezuela & Zulia & - & $10^{\circ} 00^{\prime} \mathrm{N}$ & $72^{\circ} 31^{\prime} \mathrm{W}$ & Mumaw et al. (2015) \\
\hline Venezuela & Barinas & Barinas & $08^{\circ} 37^{\prime} \mathrm{N}$ & $70^{\circ} 14^{\prime} \mathrm{W}$ & Barrio-Amorós and Ortiz (2015) \\
\hline Venezuela & Zulia & $\begin{array}{c}\text { Jardin Botânico de } \\
\text { Maracaíbo, San Francisco }\end{array}$ & $10^{\circ} 35^{\prime} \mathrm{N}$ & $71^{\circ} 42^{\prime} \mathrm{W}$ & Larreal et al. (2012) \\
\hline Venezuela & Lara & $\begin{array}{c}\text { Parque Nacional Cerro } \\
\text { Saroche }\end{array}$ & $10^{\circ} 31^{\prime} \mathrm{N}$ & $69^{\circ} 37^{\prime} \mathrm{W}$ & Suárez et al. (2013) \\
\hline Venezuela & Apure & Paez & $07^{\circ} 14^{\prime} \mathrm{N}$ & $71^{\circ} 22^{\prime} \mathrm{W}$ & Infante-Rivero (2009) \\
\hline
\end{tabular}

Carvalho 2016). The Lavrado is an ecorregion of open vegetation, covering approximately 43.281 $\mathrm{km}^{2}$ (De Carvalho and De Carvalho 2012, Carvalho et al. 2016). The municipality of Cantá presents $10.48 \%$ of its territory over lavrado areas (Morais and Carvalho 2016).

Little is known about the biodiversity of the lavrado, since these areas have been scarcely sampled in the past (Barbosa and Ferreira 2004, Flores 2014). The lavrado also lacks a specific protection within conservation units, also suffering a large anthropic pression, harboring most of the state population (Campos et al. 2008, Flores 2014). Given these circumstances, large impacts could interfere in the faunal conservation of these areas, highlighting the importance of faunal samplings and directed conservation efforts to these areas.

\section{ACKNOWLEDGMENTS}

The authors thank Conselho Nacional de Desenvolvimento Científico e Tecnológico (CNPq) for a financial aid (PIBIC Grant, 136628/2016-
8), Editor Igor Luis Kaefer and two anonymous reviewers for their suggestions to our manuscript.

\section{REFERENCES}

AB'SABER AN. 1982. The paleoclimate and paleoecology of Brazilian Amazonia. In: Prance GT (Ed), Biological Diversification in the Tropics, New York: Columbia University Press, p. 41-59.

ÁVILA-PIRES TC. 1995. Lizards of brazilian amazonia (Reptilia: Squamata). Zool Verhandelingen 299(1): 1-706.

BARBOSA RI AND FERREIRA CAC. 2004. Biomassa acima do solo de um ecossistema de "campina" em Roraima, norte da Amazônia Brasileira. Acta Amaz 34(4): 577-586.

BARBOSA RI AND LIMA CGB. 2008. Notas sobre a diversidade de plantas e fitofisionomias em Roraima através do Banco de Dados do Herbário INPA. Amaz Cien e Des 4: 131-154.

BARRIO-AMORÓS CL AND ORTIZ JC. 2015. Material herpetológico colectado por Roberto Donoso Barros em Venezuela (excepto geckos) en el Museo de Zoologia de la Universidad de Concepción, Chile. Gayana 79(1): 68-93.

BIGARELLA JJ AND ANDRADE-LIMA D.1982. Paleoenvironmental changes in Brazil. In: Prance GT (Ed), Biological Diversification in the Tropics, New York: Columbia University Press, p. 27-40.

BLANCO-TORRES A, BÁEZ SL, PATIÑO-FLORES E AND RENJIFO RJM. 2013. Herpetofauna from the middle 
valley of the Ranchería river, La Guajira, Colombia. Rev Biodivers Neotrop 3(2): 113-122.

CAMPOS C, PINTO F AND BARBOSA RI. 2008. O Lavrado de Roraima: importância biológica, desenvolvimento e conservação na maior savana do Bioma Amazônia. Ministério da Ciência e Tecnologia, Instituto Nacional de Pesquisas da Amazônia, Núcleo de Pesquisas de Roraima.

CARVALHO TM, CARVALHO CM AND MORAIS RP. 2016. Fisiografia da paisagem e aspectos biogeomorfológicos do lavrado, Roraima, Brasil. Rev Bras Geomorf 17: 93-107.

CHIPPAUX JP. 1986. Les Serpents de la Guyane française. Paris: Collection Faune Tropicale XXVII, 167 p.

COLE CJ, TOWNSEND CR, REYNOLDS RP, MACCULLOCH RD AND LATHROP A. 2013. Amphibians and reptiles of Guyana, South America: illustrated keys, annotated species accounts, and a biogeographic synopsis. P Bio Soc Wash 125(4): 317-578.

COLLI GR.1996 Amazonian savanna lizards and the biogeography of Amazonia. Ph.D. Dissertation, University of California, Los Angeles.

CUNHA OR AND NASCIMENTO FP. 1980. Ofídios da Amazônia. XI- Ofídios de Roraima e notas sobre Erythrolamprus bauperthuisii Duméril, Bibron \& Duméril, 1854, sinônimo de Erythrolamprus aesculapii aesculapii (Linnaeus, 1758). Bol Mus Para Emílio Goeldi 102: 1-2.

CUNHA OR AND NASCIMENTO FP. 1993. Ofídios da Amazônia. As cobras da região leste do Pará. Bol Mus Para Emílio Goeldi 9: 1-191.

CUNHA OR, NASCIMENTO FP AND ÁVILA-PIRES TCS. 1985. Os répteis da área de Carajás, Pará, Brasil (Testudines e Squamata). I. Pub Avulsas Mus Para Emílio Goeldi: 9-92.

DE CARVALHO TM AND DE CARVALHO CM. 2012. Interrelation of geomorphology and fauna of Lavrado region in Roraima, Brazil - suggestions for future studies. J Quat Sci 61(2): 146-155.

DUGAND A AND TOLOZA PR. 1975. Serpentifauna de la Llanura Costera del Caribe. Caldasia 11(53): 61-82.

DUNN ER.1944. Los géneros de anfibios y reptiles de Colombia, III. Orden de las serpientes. Caldasia 3(12): 155-224

EDEN MJ. 1974. Paleoclimatic influences and the development of savanna in Southern Venezuela. J Biogeogr 1: 95-109.

EITEN G. 1978. Delimitation of the Cerrado concept. Vegetatio 36: 169-178.

FLORES AS. 2014. "Os segredos das flores dos lavrados": Relato de uma ação educativa na área de Botânica no extremo norte do Brasil. Bol Mus Int Ror 8(1): 10-18.

FRANÇA FGR, MESQUITA DO AND COLLI GR. 2006. A checklist of snakes from Amazonian Savannas in Brazil, housed in the Coleção Herpetológica da Universidade de Brasília, with new distribution records. Occ Pap Sam Noble Oklahoma Mus Nat 17: 1-13.
FROTA JG, SANTOS-JR AP, CHALKIDIS HM AND GUEDES AG. 2005. As serpentes da região do baixo Rio Amazonas, Oeste do Estado do Pará, Brasil (Squamata). Biociências 13(2): 211-220.

GAIARSA MP, ALENCAR LR AND MARTINS M. 2013. Natural history of Pseudoboine snakes. Pap Avulsos Zool 53(19): 261-283.

GONZALEZ-CARACÍA JÁ, ROMERO VP AND RIVAS G. 2012. Phimophis guianensis (Troschel's Pampas Snake). Diet. Herp Rev 43(3): 496.

HOOGMOED MS. 1982. Snakes of the Guianan region. Mem Inst Butantan 46: 219-254.

HUBER O. 1982. Significance of savanna vegetation in the Amazon Territory of Venezuela. In: Prance GT (Ed), Biological diversification in the tropics. New York: Columbia University Press, p. 57-97.

INFANTE-RIVERO EE. 2009. Anfibios y Reptiles de La Guajira Venezolana. Bol Cent Investig Biol Univ Zulia 43(2): 263-277.

LANCINI AR AND KORNACKER PM.1989. Die Schlangen von Venezuela. Caracas: Armitano Editores C.A., 381 p.

LARREAL JT, RIVAS GA, PORTILLO-QUINTERO C AND BARROS TR. 2012. Squamata reptiles of a fragment of tropical dry forest in northwestern Venezuela (Lake Maracaibo region). Check List 8(6): 1220-1224.

MORAIS RP AND DE CARVALHO TM. 2016. Aspectos dinâmicos da paisagem do lavrado, nordeste de Roraima. Geociências 34(1): 55-68.

MUMAW MN, GONZÁLEZ LFE AND FERNÁNDEZ MC. 2015. Atlas Serpientes de Venezuela: Uma Visón Actual de su Diversidad. Santiago de Chile: Dimacofi Negocios Avanzados S.A., 462 p.

PETERS JA, OREJAS-MIRANDA, B AND DONOSOBARROS R. 1970. Catalogue of the Neotropical Squamata: Lizards and Amphisbaenians (No. 297). Smithsonian Institution Press.

RAY JM AND RUBACK P. 2015. Updated checklists of snakes for the provinces of Panamá and Panamá Oeste, Republic of Panama. Mesoam Herpetol 2(2): 168-188.

SAWAYA RJ, MARQUES OAV AND MARTINS M. 2008. Composição e história natural das serpentes de Cerrado de Itirapina, São Paulo, sudeste do Brasil. Biota Neotrop 8: 127-149.

STARACE F. 1998. Guide des Serpents et Amphisbènes de Guyane. Cayenne: IBIS Rouge Editions, 450 p.

SUÁREZ CLV, RODRÍGUES JGV, PEÑA FER AND TOJEIRO YSM. 2013. Lista actualizada y distribución espacial de la riqueza de anfibios y reptiles del Parque Nacional Cerro Saroche, Estado Lara, Venezuela. Ecotrópicos 26(1-2): 40-54.

THOMAS RA. 1976. A revision of the South American colubrid snake Philodryas Wagler, 1830. Doctoral 
Dissertation, Texas A\&M University College Station. (Unpublished).

TROSCHEL FH. 1848. Amphibien. In: M.R. Schomburgk. Reisen in Britisch-Guiana in den Jahren 1840-44. Im Auftrage Sr. Majestät des Königs von Preussen ausgeführt. Theil 3. Versuch einer Zusammenstellung der Fauna und Flora von British-Guiana. Leipzig, p. 645-661.

UETZ P, FREED P AND JIRÍ HOŠEK. 2017. The Reptile Database, http://www.reptile-database.org, accessed at 05/02/2018.

VANZOLINI PE. 1986. Levantamento Herpetológico da Área do Estado de Rondônia sob Influência da Rodovia BR 364. Brasília, Distrito Federal, Brasil, Conselho Nacional do Desenvolvimento Científico e Tecnológico, Assessoria Editorial.
VANZOLINI PE AND CALLEFFO MEV. 2002. A taxonomic bibliography of the South American snakes of the Crotalus durissus complex (Serpentes, Viperidae). An Acad Bras Cienc 74: 37-83.

VITT LJ AND DE CARVALHO CM. 1995. Niche partitioning in a tropical wet season: lizards in the lavrado area of northern Brazil. Copeia 305-329.

YANOSKY A, DIXON J AND MERCOLLI C. 1996. Ecology of a snake community of El Bagual Reserve, Argentina. Herpetol Nat Hist 4: 97-110.

ZAHER H. 1996. A new genus and species of pseudoboine snakes, with a revision of the genus Clelia (Serpentes, Xenodontinae). Boll Mus Regionale Sci Nat Torino 14(2): 289-337. 